\title{
INTEGRATION OF BUILDING INFORMATION MODELING AND AGENT-BASED MODELING FOR EVACUATION SIMULATION
}

\author{
C. Beyaz ${ }^{\mathrm{a}}$, E. D. Özgener ${ }^{\mathrm{a}}$, Y. G. Bağc1 ${ }^{\mathrm{a}}$, Ö. $\mathrm{Akın}^{\mathrm{a}}{ }^{\text {, H. Demirel }}{ }^{\mathrm{a}}$ \\ ${ }^{a}$ Istanbul Technical University Civil Engineering Faculty, Istanbul, Turkey
}

KEY WORDS: Building Information Modeling, Agent-Based Modeling, Evacuation, Risk Assessment, Simulation, 3D

\begin{abstract}
:
Building Information Modelling (BIM) is a highly advanced spatial modeling method that is fully incorporated in the building lifecycle. With the support of Information Technologies, the use of BIM has become common in building management such as energy efficiency, indoor navigation and emergency evacuation simulations. This study focuses on emergency evacuation simulations since, integrating BIM and Spatial Information Science, could mitigate casualties in emergencies. Traditional evacuation management methods are generally inadequate since they are based on $2 \mathrm{D}$ evacuation plans, they are static and do not consider the characteristics/interactions of the people in the building. This study aims to integrate BIM and Agent-Based Modelling (ABM) for emergency evacuation simulations, where characteristics of the building and the users are incorporated. Istanbul Technical University Faculty of Civil Engineering was selected as study area and the BIM model was created by using the CAD drawings of the floor plans. The users of the Faculty building such as students, academicians, administrative staff and visitors are considered for simulations. The BIM model was transferred to the ABM environment, and the routes used during the fire evacuation were generated. Fire evacuation simulations were performed, where agents having different characteristics evacuate the building according to the rules predefined. Three different scenarios were tested. Major conclusion of this study is that, via integrating BIM and ABM, it is possible to model people's behavior within a three-dimensional digital environment, where decision-makers could be performing simulations such as fire evacuation supported by dynamic, realistic and accurate information.
\end{abstract}

\section{INTRODUCTION}

A high percentage of emergency casualties are directly related to the facility's delayed evacuation service (Wang et al., 2017). Especially in crowded public areas such as schools, hospitals, and shopping malls, loss of life and properties can be severe if inadequate and improper emergency evacuation planning is undertaken. Traditional evacuation management methods are generally inadequate since they are based on $2 \mathrm{D}$ evacuation plans and do not consider the characteristics/interactions of the people in the building. In addition, since people know that they are not in a dangerous situation during evacuation studies and exercises, they do not fully reflect the situation in the real evacuation (Zhang and Issa, 2015). Considering the behavior of people during a moment that causes panic, the losses become significantly damaging. To better manage the situation, digital simulation environments that demonstrate and testify building evacuation plans should be generated for enhancing the decision-making process in evacuation scenarios (Tang and Ren, 2011).

Agent-Based Modeling (ABM) is a simulation technique that allows to modeling of individual properties and interactions that directly affect the dynamic behavior of the whole system (Stanilov, 2011). The ABM approach consists of an organized set of agents that can interact with each other in a virtual world (Naili and Bourahla, 2019). Especially for the systems where the most complex dynamics are driven by human behaviors, it is crucial to understand and model these individual properties to evaluate the outcomes in the real world. Human behaviors and interactions with each other can be modeled through the agent paradigm for evacuation scenarios. During a disaster event, as an example, obstacles may occur on the exit routes and the routes closest to the exit point may change dynamically. Therefore, not only interactions of people, but also the effect of the environment must be modeled in such studies. With the help of advanced spatial modeling techniques such as Building Information Modeling (BIM), the spatial properties of an event could be integrated into the modeling environment in their original form. Linking BIM with $\mathrm{ABM}$ is widely used in evacuation studies to obtain realistic and dynamic simulation results to better manage and support disaster management processes (Tan et al., 2015; Kasereka et al., 2018; Sun and Turkan, 2020).

This study aims to model and observe the behaviors and interactions of the agents in a three-dimensional building in case of an emergency evacuation. For this simulation, rich semantic information in BIM models and an ABM approach controlling human behavior were integrated. It is feasible to represent people's behavior within a three-dimensional digital environment using BIM and ABM, with decision-makers executing simulations such as fire evacuation utilizing dynamic, realistic, and accurate data. The simulation was performed in the Istanbul Technical University Faculty of Civil Engineering building where several different profiles exist.

\section{SYSTEM ARCHITECTURE}

The system architecture of the study is illustrated in Figure 1. Firstly, two-dimensional CAD drawings of Istanbul Technical University, Faculty of Civil Engineering building were generated as three-dimensional BIM, where Autodesk Revit software was utilized. The detailed BIM model of the faculty is generated by considering the features. 
The BIM model was exported to the Unity platform by using the Twin Motion plug-in and the topology was created using the properties of the features. After that, an Indoor navigational surface/network was generated by using the generated topology. $\mathrm{ABM}$ approach was used to simulate the actions and interactions of agents (both individual and collective entities such as organizations or groups) to make predictions about the behavior of the system and to understand what governs its results. The number of people in the faculty, accessible areas and obstacles were determined using the algorithms within Unity and C\# programming language.

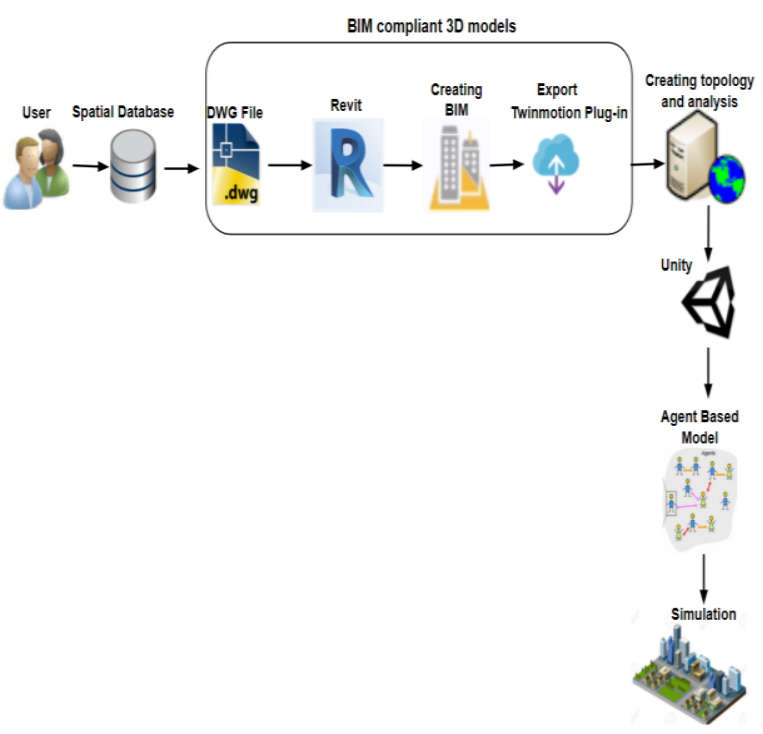

Figure 1. The designed frame-work

\section{DATA \& METHODOLOGY}

\subsection{Data and Study Area}

Istanbul Technical University (ITU) Civil Engineering Faculty Building was chosen as the study area of the study as it consists of people having different responsibilities and profiles and has a complex building structure. Two dimensional CAD drawings of the building were used to generate a BIM model of the faculty in Revit software. These drawings contain structures such as windows, stairs, doors, and columns in two dimensions. To generate a three-dimensional model, 2D CAD drawings were converted into a 3D Building Information Model by adding semantic information of the building objects. Then the generated model was transferred to the environment where the $\mathrm{ABM}$ approach was used. In the ABM environment, the visual quality of generated model has been enriched by adding outdoor elements to the environment. The final stage of the simulation environment is illustrated in Figure 2.

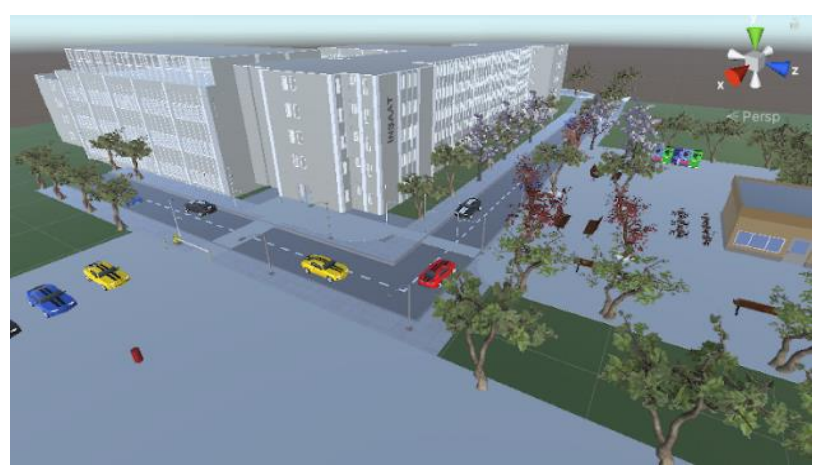

Figure 2. Visualization of Faculty building

\subsection{Methodology}

\subsubsection{Integration of BIM \& ABM}

Since the interaction between agents and the environment needs to be simulated, it is necessary to provide a three-dimensional environment in high granularity to use the agent-based approach. The decision/action functions of the agents will vary according to their individual characteristics, responsibilities and interactions with each other and the environment. For using the ABM approach, Unity software is selected as a simulation platform. Unity is a game engine developed by Unity Technologies and the engine is used to create threedimensional, two-dimensional, virtual reality, and augmented reality games, as well as simulations and other experiences. After the algorithms were generated in the BIM model, the evacuation simulation was deployed.

In the evacuation simulations, modeling the dynamic behavior of people has significant importance. These behaviors are usually shaped by what people see. At this point, the need of including visibility analyzes that reveal the coverage area of people's view in the evacuation simulations increases significantly. Figure 3 shows the visible zone in green and its cross-section with the eye level height of an agent (i.e., visibility area) in blue.

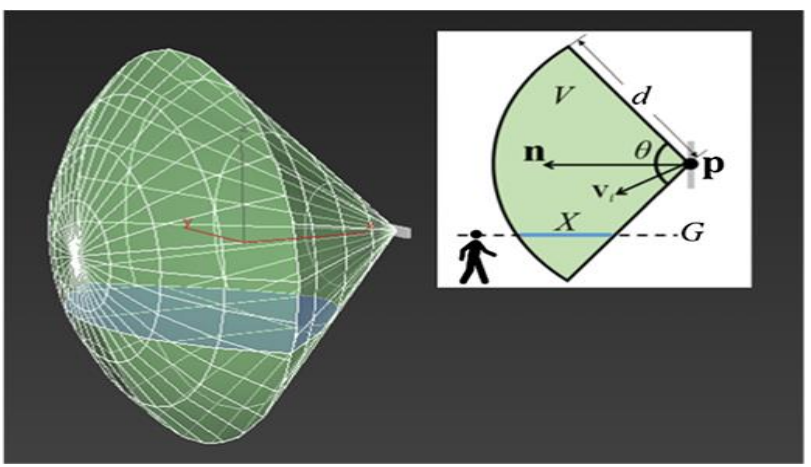

Figure 3. Visibility algorithm in Unity Representation (Motamedi et al., 2017).

\section{RESULTS}

The study generated a very realistic and detailed digital environment to analyze evacuation scenarios in case of a fire disaster. In this context, emergency escape plans were created and analyzed, where visibility and accessibility algorithms were performed for the evacuation of people with different profiles.

Agents placed in the faculty are intended to imitate human characteristics. Building environment and human behavior are the two critical scenario variables in real-world building evacuation. Focusing on these two variables, the essential task of evacuation simulation is to represent the evacuee's interaction with the building environment (Cheng et al., 2018). For this reason, it is necessary to divide the people in the building into groups and determine their characteristics. When the ITU construction faculty is examined, there are 4 basic groups of people, these are; Students, Academic staff, Management unit and permanent workers. The character properties and subgroups of the agents are shown in Figure 4.

Different speeds and behaviors were defined for agents according to each group and simulations were carried out. In 
addition, during the evacuation, people could recognize obstacles via the visibility algorithm. Agents move towards the determined target destination (assembly area) through the generated navigation networks. The Faculty of Civil Engineering has several exit doors, and the evacuation of the agents was simulated towards the nearest exit according to their initial position. Furthermore, evacuation performance such as evacuation time, speed of agents and others were determined.

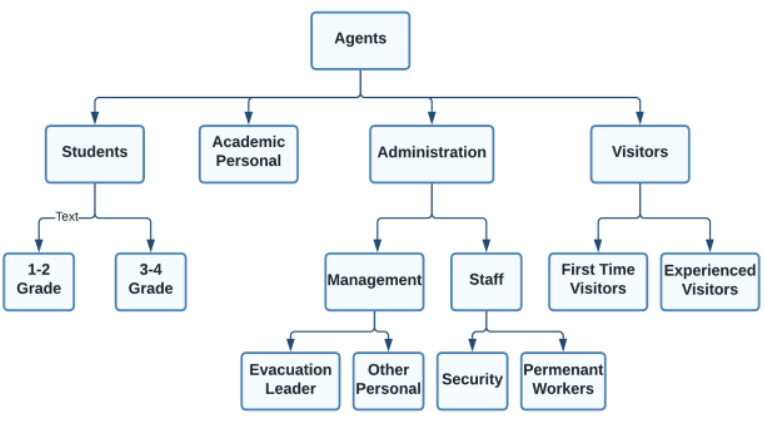

Figure 4. Agents Groups

The agents in the scenario were created according to the characteristics of the people in the faculty. Due to the possibility of some agents having a crisis during the evacuation, the characters are given panic features. In addition, disabled people can be found at the time of the fire. It is expected that every agent has different familiarity with the building and may behave differently during evacuation (Bercha F., 2014). For this reason, it has been examined in 2 different experience levels. Students are separated among themselves, 3-4 graders head directly to the door, 1-2 graders follow the exit signs and head for the exit. The academic staff waits for everyone from the classroom to evacuate and helps the panicked agents. First-time visitors do not know the location of the exit door directly. Therefore, first time visitors only have the characteristic to exit using visibility.

To generate the realistic simulation platform, navigation networks were created according to the building topology. A component called "NavMesh" has been added to the simulation platform so that agents can act on the created network. The NavMesh component calculates the shortest path that the selected object could move from an origin to the determined destination. After the relevant parameters were entered in the navigation window, the navigation surfaces for the agents are obtained. In Figure 5, the simulation environment, navigation surface and the sample agents were presented. A simulation was created for crowd behavior, which includes examining how groups move and avoid collisions in such environments in case of a fire in the faculty, as illustrated in Figure 6.

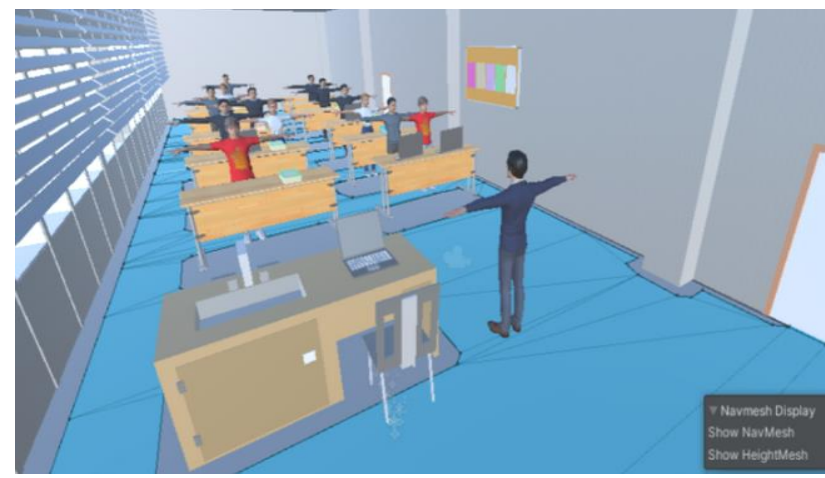

Figure 5. Simulation environment and the navigation surface

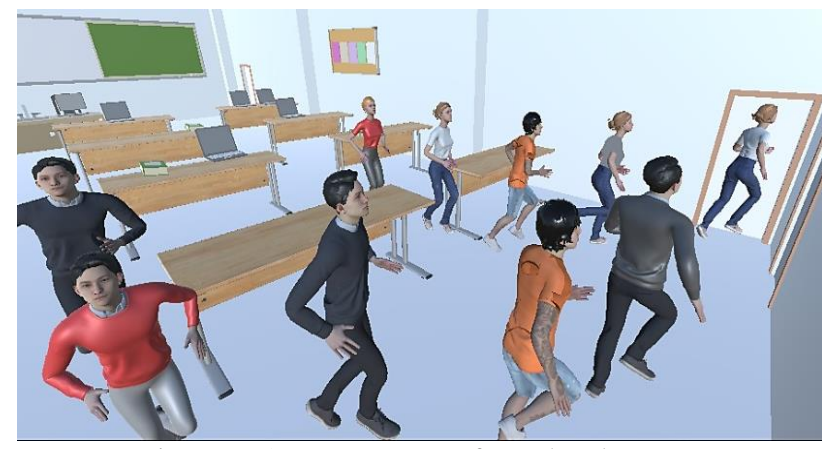

Figure 6. Agents runaway from the classroom

Running motion was added to the characters and their animations were created. To make the movements of the characters more realistic, they were allowed to turn their heads towards the direction they are headed. At this stage, the body part of the script characters created using the $\mathrm{C \#}$ programming language was cut and placed in the head part, where was illustrated in Figure 7.

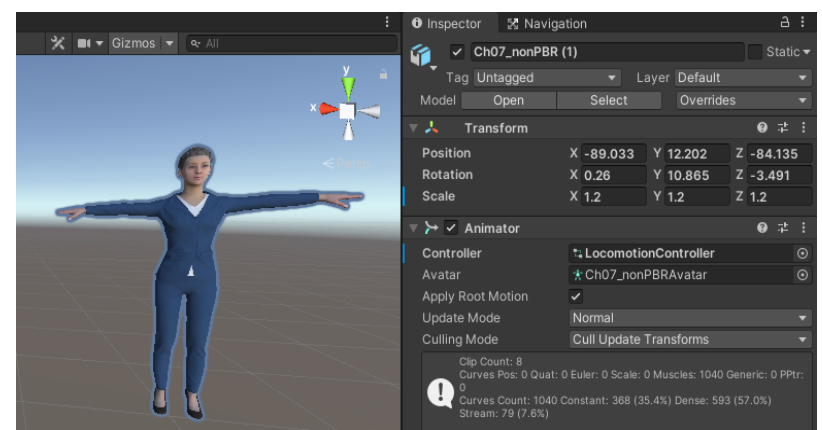

Figure 7. More realistic agents

This visibility function has been integrated into agents and agents were provided to see a certain feature such as an exit sign or other people. This view of the agents was primarily used during the fire simulation, for the 1-2th graders and visitors, who are described as "1-2 level inexperienced", to see the exit sign and move in line with the signs. In this way, the possible movements and behaviors of agents during the fire were realistically modeled as illustrated in Figure 8. During the fire event, the agents need to escape from that area by displaying realistic behaviors when they see the fire. Within the scope of the study, a visibility zone was created, and the agents who saw the fire during the fire simulation were allowed to turn to the other side where there was no fire and leave the faculty from that exit instead of the nearest. In this way, when the agents enter the field of view of the fire just like people, they escape and the duration of the evacuation and the exit points change.

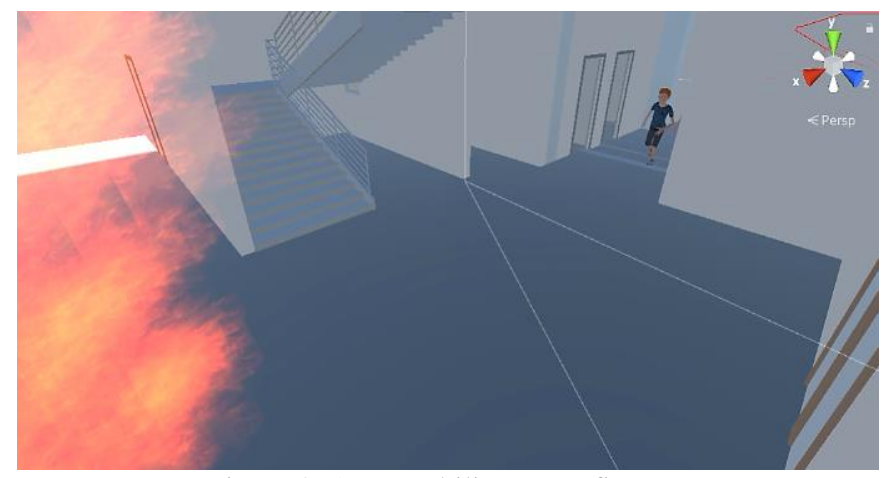

Figure 8. Agents ability to see fire 
Furthermore, as stated by Ren et al.(2017), one of the people's most common behavior during a fire is panic. Within the scope of the study, for each evacuated class, some agents exposed the panic and needed another agent for evacuation, as illustrated in Figure 9. Panic agents wait until another agent comes to their side and act only with the agent who comes to them. In this way, the scenario in which some people panic and remain inactive during the fire and need serious help, as in real life, has been integrated into fire simulation. The panicked person and the person supporting him or her are the last to leave the classroom, and the elapsed time affects the evacuation time. During the study integration, the scenario of students panicking and academicians helping was processed, via prepared C\# script. Hence, it is possible to integrate this panic situation with an agent.

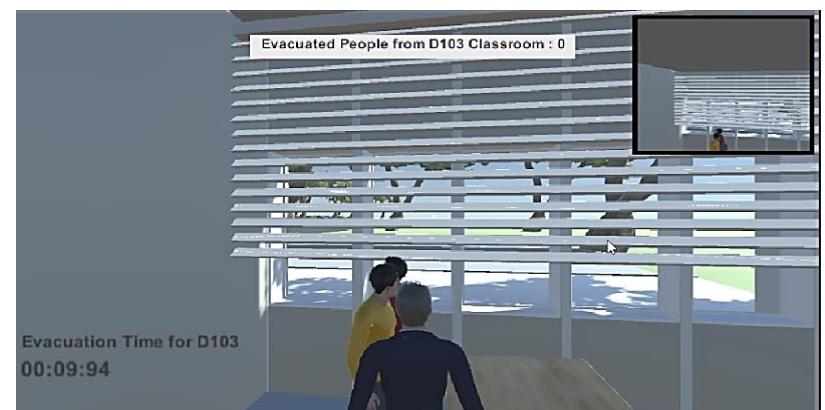

Figure 9. The arrival of Lecturer next to Panic Agent

Within the scope of the study, it was aimed to create a fire evacuation simulation for the faculty. The simulation was performed in three different ways due to insufficient hardware performance. First, an evacuation simulation of the entire faculty was carried out using cylindrical agents. Then a single floor simulation has performed using uniform agents and without factors affecting hardware performance such as faculty furniture and outdoor elements. Finally, classroom-based evacuation simulations were performed using multiple agents, faculty furniture and outdoor elements, and evacuation times were generated for each scenario.

In the first scenario where cylindrical agents were used, 480 agents were used for all floors of the faculty and thanks to the navigation surface created, the agents moved towards the shortest exit in case of fire and evacuated the faculty. In this scenario, the total evacuation took 3 minutes. In Scenario 2, an evacuation simulation was performed for a single floor of the faculty using uniform agents. In this scenario, attention was paid to factors such as some agents panicking, some being disabled, some seeing the fire and heading for the other exit. As a result of these situations, the total evacuation took a minute. In the last scenario, class-based evacuation simulations were carried out for 4 classes by using different types of agents and faculty furniture and external elements. To obtain realistic and accurate analysis results within the scope of the study, different speeds were used according to the types of characters and simulation was carried out. According to the results obtained, it was analyzed that the D101 classes had the shortest evacuation time with 28 seconds, and the D103 classes with the longest evacuation time 35 seconds.

\section{CONCLUSION}

By integrating BIM and ABM, it is possible to model people's relationships with each other and the environment inside of the building. Consequently, more accurate emergency evacuation simulation has been achieved. Evaluating and modeling the evacuation process in a $3 \mathrm{D}$ environment correctly manages the decision-making process at the time of evacuation and also creates ideas for decision-makers. As in this study, disaster evacuation scenarios and simulations could be performed in an digital realistic environment via the integration of theoretical and practical knowledge brought by multiple disciplines.

\section{REFERENCES}

Cheng, J. C. P., Tan, Y., Song, Y., Mei, Z., Gan, V. J. L., and Wang, X., 2018. Developing an evacuation evaluation model for offshore oil and gas platforms using BIM and agent-based models. Automation in Construction, 89, 214224.

Kasereka, S., Kasoro, N., Kyamakya, K., Goufo, E.F.D., Chokki, A.P., and Yengo, M.V., 2018. Agent-Based Modelling and Simulation for evacuation of people from a building in case of fire. Procedia Computer Science, 130, 10-17.

Motamedi, A., Wang, Z., Yabuki, N., Fukuda, T., and Michikawa, T., 2017. Signage visibility analysis and optimization system using BIM-enabled virtual reality (VR) environments. Advanced Engineering Informatics, 32, 248262.

Naili, M., and Bourahla, M., 2019. Stability-based model for evacuation system using agent-based social simulation and Monte Carlo method. International Journal of Simulation and Process Modelling, Vol. 14, no. 1, pp. 1-16.

Ren C., Yang C., and Jin S., 2009. Agent-Based Modeling and Simulation on Emergency Evacuation. Complex 2009, Part II, LNICST 5, pp. 1451 - 1461.

Stanilov, K., 2011. Space in agent-based models. Agent-Based Models of Geographical Systems, 253-269.

Sun, Q., and Turkan, Y., 2020. A BIM-based simulation framework for fire safety management and investigation of the critical factors affecting human evacuation performance. Advanced Engineering Informatics, 44, 101093.

Tan, L., Hu, M., and Lin, H., 2015. Agent-based simulation of building evacuation: Combining human behavior with predictable spatial accessibility in a fire emergency. Information Sciences, 295, 53-66.

Tang, F., and Ren, A., 2011.GIS-based 3D evacuation simulation for indoor fire. Building and Environment, 193-202

Wang Z., Kim B., Kobayashi H., Wu G., and Barth M. J., 2018. Agent-Based Modeling and Simulation of Connected and Automated Vehicles Using Game Engine: A Cooperative OnRamp Merging Study. ArXiv, 1810.09952

Wang Z., Yang H., and Zhu Z., 2017. Development of A Simulation Model for Pedestrian Evacuation Under Fire Condition. Society for Modeling Simulation International, 14, $1-11$.

Zhang, J., and Issa, R.R., 2015. Collecting fire evacuation performance data using BIM-based immersive serious games for performance-based fire safety design. Computing in Civil Engineering, 612-619. 